Internat. J. Math. \& Math. Sci.

Vol. 23, No. 12 (2000) 819-825

S0161171200003082

(c) Hindawi Publishing Corp.

\title{
CLASSES OF CONVEX FUNCTIONS
}

\section{THOMAS ROSY, B. ADOLF STEPHEN, K. G. SUBRAMANIAN, and HERB SILVERMAN}

(Received 1 March 1999)

ABSTRACT. We investigate a family that connects various subclasses of functions convex in the unit disk. We also look at generalized sequences for this family.

Keywords and phrases. Convex functions.

2000 Mathematics Subject Classification. Primary 30C45.

1. Introduction. Denote by $S$ the family of functions

$$
f(z)=z+\sum_{k=2}^{\infty} a_{k} z^{k}
$$

that are analytic and univalent in the unit disk $\Delta=\{z:|z|<1\}$ and by $K$ the family of convex functions $f \in S$ for which $\operatorname{Re}\left(1+z f^{\prime \prime} / f^{\prime}\right)>0, z \in \Delta$. There are several well-known subclasses of $K$. Robertson in [6] introduced the family $K(\alpha)$ of functions $f$ convex of order $\alpha, 0 \leq \alpha<1$, that satisfy in $\Delta$ the inequality $\operatorname{Re}\left(1+z f^{\prime \prime} / f^{\prime}\right)>\alpha$. Ruscheweyh [8] defined the subclass $D$ of $K$ consisting of functions $f$ for which $\operatorname{Re} f^{\prime}(z) \geq\left|z f^{\prime \prime}(z)\right|, z \in \Delta$. His convolution conjecture [8] for this class is stronger than the (former) Bieberbach conjecture (deBranges' theorem).

Goodman [2] introduced the family UCV $\subset K$ of uniformly convex functions $f$ having the property that for every circular arc $\gamma$ contained in $\Delta$ with center also in $\Delta$, the image $\operatorname{arc} f(\gamma)$ is a convex arc. He then gave the two-variable characterization

$$
\operatorname{Re}\left[1+\frac{(z-\zeta) f^{\prime \prime}(z)}{f^{\prime}(z)}\right]>0, \quad(z, \zeta) \in \Delta \times \Delta
$$

Ma and Minda [4] and Ronning [7] independently found a more applicable one-variable characterization for UCV, namely

$$
\operatorname{Re}\left[1+\frac{z f^{\prime \prime}(z)}{f^{\prime}(z)}\right] \geq\left|\frac{z f^{\prime \prime}(z)}{f^{\prime}(z)}\right|, \quad z \in \Delta .
$$

We may summarize relationships between $K(\alpha), D$, and UCV.

THEOREM 1.1. (i) $D \not \subset K(\alpha), \alpha>0 ; K(\alpha) \not \subset D, \alpha<1$.

(ii) $D \not \subset \mathrm{UCV}$ and UCV $\not \subset D$.

(iii) $\mathrm{UCV} \subset K(1 / 2)$. See [7]. 
Proof OF (i). The function $z+z^{2} / 4 \in D-K(\alpha), \alpha>0$, and

$$
\int_{0}^{z}(1-t)^{-2(1-\alpha)} d t \in K(\alpha)-D, \quad \alpha<1
$$

Proof OF (ii). For $z+a_{2} z^{2}+a_{3} z^{3}+\cdots \in D$ and $z+b_{2} z^{2}+b_{3} z^{3}+\cdots \in \mathrm{UCV}$, the sharp coefficient bounds $\left|a_{2}\right| \leq A_{2}=\sqrt{2}-1$ and $\left|a_{3}\right| \leq A_{3}=2 / 3(\sqrt{5}-2)$ were found in [1], while $\left|b_{2}\right| \leq B_{2}=4 / \pi^{2}$ and $\left|b_{3}\right| \leq B_{3}=8 / 9 \pi^{2}+32 / 3 \pi^{4}$ were found in [4]. Since $A_{2}>B_{2}$ and $B_{3}>A_{3}$, neither inclusion is possible.

In this paper, we introduce a family of functions that connects these various subclasses of $K$. We also relate this new class to the family $R$ of functions $f \in S$ for which $\operatorname{Re} f^{\prime}>0, z \in \Delta$.

2. The main class. We say that $f$ of the form (1.1) is in $\operatorname{UCD}(\alpha), \alpha \geq 0$, if

$$
\operatorname{Re} f^{\prime}(z) \geq \alpha\left|z f^{\prime \prime}(z)\right|, \quad z \in \Delta .
$$

Note that $\operatorname{UCD}(0)=R$ and $\operatorname{UCD}(1)=D$. Note further that $\operatorname{UCD}(\alpha) \not \subset S$ if $\alpha<0$, since $z+(1-\alpha) z^{2} / 2 \in \mathrm{UCD}(\alpha)-S, \alpha<0$.

THEOREM 2.1. $\operatorname{UCD}(\alpha) \subset K(1-1 / \alpha), \alpha \geq 1$, and the result is sharp.

Proof. If $f \in \operatorname{UCD}(\alpha)$, then

$$
\left|f^{\prime}(z)\right| \geq \alpha\left|z f^{\prime \prime}(z)\right|, \quad\left|\frac{z f^{\prime \prime}(z)}{f^{\prime}(z)}\right| \leq \frac{1}{\alpha} .
$$

Hence,

$$
\operatorname{Re}\left[1+\frac{z f^{\prime \prime}(z)}{f^{\prime}(z)}\right] \geq 1-\left|\frac{z f^{\prime \prime}(z)}{f^{\prime}(z)}\right| \geq 1-\frac{1}{\alpha} .
$$

For sharpness, set $f(z)=\int_{0}^{z}((1+c t) /(1-c t)) d t, c=\sqrt{1+\alpha^{2}}-\alpha$. Then $f \in \operatorname{UCD}(\alpha)$ because for $|z|=r<1, \operatorname{Re} f^{\prime}(z)=\left(1-c^{2} r^{2}\right) /\left(|1-c z|^{2}\right) \geq \alpha(2 c r) /\left(|1-c z|^{2}\right)=$ $\alpha\left|z f^{\prime \prime}(z)\right|$. Note that $\operatorname{Re}\left[1+z f^{\prime \prime} / f^{\prime}\right]=\operatorname{Re}\left[1+2 c z /\left(1-c^{2} z^{2}\right)\right]$. For $z=-r, r \rightarrow 1$, this last expression approaches $1-2 c /\left(1-c^{2}\right)=1-1 / \alpha$. Thus, $f \notin K(\beta)$ for $\beta>1-1 / \alpha$.

Clearly, the family $\operatorname{UCD}(\alpha) \subset D$ for $\alpha \geq 1$. We next see when $\operatorname{UCD}(\alpha)$ is uniformly convex.

THEOREM 2.2. $\mathrm{UCD}(\alpha) \subset \mathrm{UCV} \Leftrightarrow \alpha \geq 2$.

Proof. Since the extremal function of Theorem 2.1 is not in $K(1 / 2)$ for $\alpha<2$, an application of Theorem 1.1(iii) shows that this function cannot be in UCV when $\alpha<2$. If $f \in \mathrm{UCD}(2)$, then

$$
\left|f^{\prime}(z)\right| \geq 2\left|z f^{\prime \prime}(z)\right|, \quad\left|\frac{z f^{\prime \prime}}{f^{\prime}}\right| \leq \frac{1}{2} .
$$

Thus,

$$
\operatorname{Re}\left[1+\frac{z f^{\prime \prime}(z)}{f^{\prime}(z)}\right] \geq 1-\left|\frac{z f^{\prime \prime}}{f^{\prime}}\right| \geq\left|\frac{z f^{\prime \prime}}{f^{\prime}}\right|, \quad f \in \mathrm{UCV}
$$


3. Sequences. To a finite or infinite increasing sequence of integers $\left\{n_{k}\right\}$ with $n_{k} \geq k$ we associate with $f$ of the form (1.1) the generalized partial sum defined by

$$
\tilde{f}(z)=z+\sum_{k=2}^{\infty} a_{n_{k}} z^{n_{k}},
$$

with the special case $n_{k}=k(k=2,3, \ldots, n)$ representing the $n$th section $f_{n}(z)=$ $z+\sum_{k=2}^{n} a_{k} z^{k}$. We determine when generalized sequences of functions in $R$ satisfy conditions to be in $\operatorname{UCD}(\alpha)$. Since our results rely on properties for continuous linear functionals defined on $R$, sharp results are obtained from the extreme points of $R$. See [3]. It thus suffices to consider the extremal function $f \in R$ defined by

$$
f(z)=-z-2 \log (1-z)=z+2 \sum_{k=2}^{\infty} \frac{z^{k}}{k} .
$$

In [10] it was shown for $f \in R$ that

(i) $4 f_{n}(z / 4) \subset D$,

(ii) $f(a z) / a \subset D, a=\sqrt{2}-1$.

The proof of (i) for $|z|=r \leq 1 / 2$ relied on the inequalities

$$
\operatorname{Re} f_{n}^{\prime}(z) \geq \frac{(1+r)^{2}(1-2 r)}{|1-z|^{2}}, \quad\left|f_{n}^{\prime \prime}(z)\right| \leq \frac{2(1+r)^{2}}{|1-z|^{2}},
$$

and of (ii) for $r<1$ on

$$
\operatorname{Re} f^{\prime}(z) \geq \frac{\left(1-r^{2}\right)}{|1-z|^{2}}, \quad\left|f^{\prime \prime}(z)\right| \leq \frac{2}{|1-z|^{2}} .
$$

We extend these results to the class $\operatorname{UCD}(\alpha)$.

THEOREM 3.1. If $f \in R$, then

(i) $f_{n}(b z) / b \in \operatorname{UCD}(\alpha), b=1 / 2(1+\alpha)$,

(ii) $f(a z) / a \in \operatorname{UCD}(\alpha), a=\sqrt{\alpha^{2}+1}-\alpha$.

The results are sharp for all $\alpha \geq 0$.

Proof of (i). From (3.3) we have

$$
\operatorname{Re} f_{n}^{\prime}(z) \geq \alpha\left|z f_{n}^{\prime \prime}(z)\right| \quad \text { when }(1+r)^{2}(1-2 r) \geq 2 \alpha r(1+r)^{2},
$$

which is true for $r \leq 1 / 2(1+\alpha)$. Equality holds for $f$ defined by (3.2) and $n=2$.

Proof of (ii). From (3.4) we see that

$$
\operatorname{Re} f^{\prime}(z) \geq \alpha\left|z f^{\prime \prime}(z)\right| \text { when } 1-r^{2} \geq 2 \alpha r
$$

which holds for $r=\sqrt{\alpha^{2}+1}-\alpha$.

REMARK 3.2. The case $\alpha=0$ in (i) $(f \in R)$ is due to MacGregor [5].

We now turn to generalized sequences. 
THEOREM 3.3. If $f$ of the form (1.1) is in $R$ with $\tilde{f}$ of the form (3.1) a generalized sum of $f$, then $\tilde{f}(b z) / b \in \operatorname{UCD}(\alpha), \alpha \geq 0$, where $b$ is the positive zero in $(0,1)$ of

$$
1-2 r-r^{2}-2 \alpha r \frac{1+r^{2}}{1-r^{2}}=0
$$

The result is sharp for all $\alpha$.

REMARK 3.4. The cases $\alpha=0(b=\sqrt{2}-1)$ and $\alpha=1(b \approx 0.2253)$ were proved in [10]. Note that the value for $b$ decreases as $\alpha$ increases.

Proof. We need only consider $f$ defined by (3.2). Defining $h$ by

$$
h^{\prime}(z)=f^{\prime}(z)+\alpha e^{i \gamma} z f^{\prime \prime}(z), \quad \gamma \text { real, }
$$

it suffices to show that

$$
\tilde{h}^{\prime}(z)=\tilde{f}^{\prime}(z)+\alpha e^{i \gamma} z \tilde{f}^{\prime \prime}(z)=1+2 \sum_{n_{k}=2}^{\infty}\left[1+\alpha\left(n_{k}-1\right) e^{i \gamma}\right] z^{n_{k}-1}
$$

has positive real part for $|z|<b$. We examine different cases.

CASE $1\left(n_{2} \geq 3\right)$. Then

$$
\begin{gathered}
\operatorname{Re} \tilde{h}^{\prime}(z) \geq 1-2 \sum_{n=3}^{\infty}[1+\alpha(n-1)] r^{n-1}=1-\frac{2 r^{2}}{1-r}-\frac{2 \alpha r^{2}(2-r)}{(1-r)^{2}}, \\
(1-r)^{2} \operatorname{Re} \tilde{h}^{\prime}(z) \geq 1-2 r-r^{2}-2 \alpha r^{2}(2-r) \geq 1-2 r-r^{2}-2 \alpha r
\end{gathered}
$$

Since this last expression is bounded below by the left-hand side of (3.7), it follows that

$$
\operatorname{Re} \tilde{h}^{\prime}(z) \geq 0 \text { for }|z| \leq b
$$

CASE $2\left(n_{2}=2, n_{3}=3\right)$. Then for $z=r e^{i \theta}$,

$$
\begin{aligned}
\operatorname{Re} \tilde{h}^{\prime}(z) & \geq \operatorname{Re}\left[1+2\left(1+\alpha e^{i \gamma}\right) z+2\left(1+2 \alpha e^{i \gamma}\right) z^{2}\right]-2 \sum_{n=4}^{\infty}[1+\alpha(n-1)] r^{n-1} \\
& :=\operatorname{Re} A(z)-\frac{2 r^{3}}{(1-r)^{2}}[(1-r)+\alpha(3-4 r)]
\end{aligned}
$$

Now

$$
\begin{aligned}
\operatorname{Re} A(z) & =1+2 r \cos \theta+2 r^{2} \cos 2 \theta+\operatorname{Re}\left[2 \alpha e^{i \gamma}\left(z+2 z^{2}\right)\right] \\
& \geq 1+2 r \cos \theta+2 r^{2} \cos 2 \theta-2 \alpha r(1+2 r \cos \theta)
\end{aligned}
$$

which attains its minimum for $r=b$ when $\cos \theta=-(1-2 \alpha b) / 4 b$. Thus,

$$
\begin{aligned}
& \operatorname{Re} A(z) \geq \frac{3}{4}-2 b^{2}-\alpha b-\alpha^{2} b^{2} \text { for }|z| \leq b \\
& \operatorname{Re} \tilde{h}^{\prime}(z) \geq \frac{3}{4}-2 b^{2}-\alpha b-\alpha^{2} b^{2}-\frac{2 b^{3}}{(1-b)^{2}}(1-b+\alpha(3-4 b)) .
\end{aligned}
$$


Substituting from (3.7) the value $\alpha=\left(1-b^{2}\right)\left(1-b^{2}-2 b\right) / 2 b\left(1+b^{2}\right)$ into the righthand side of (3.14), one can show that the right-hand side of (3.14) decreases as $b$ decreases. Since $\alpha b \rightarrow 1 / 2$ as $\alpha \rightarrow \infty$, we see that $\operatorname{Re} \tilde{h}^{\prime}(z) \geq 3 / 4-1 / 2-1 / 4=0$, $|z| \leq b$.

When $n_{2}=2$ and $n_{3} \geq 4$, we consider two remaining possibilities, depending on whether the first $n_{k}$ after consecutive even integers is the succeeding odd integer.

CASE 3. We have

$$
\begin{aligned}
\tilde{h}^{\prime}(z)= & 1+2 \sum_{n=1}^{m+1}\left[1+\alpha(2 n+1) e^{i \gamma}\right] z^{2 n-1}+2\left[1+\alpha(2 m+2) e^{i \gamma}\right] z^{2 m+2} \\
& +2 \sum_{n_{k} \geq 2 m+4}\left[1+\alpha\left(n_{k}-1\right) e^{i \gamma}\right] z^{n_{k}-1}
\end{aligned}
$$

Setting $r^{\prime}(z)=h^{\prime}(z)-\tilde{h}^{\prime}(z)$, we have for $|z| \leq b$ that

$$
\begin{aligned}
\operatorname{Re} \tilde{h}^{\prime}(z) & \geq \frac{1-b^{2}-2 \alpha b}{(1+b)^{2}}-\left|r^{\prime}(z)\right| \\
& \geq \frac{1-b^{2}-2 \alpha b}{(1+b)^{2}}-2 \sum_{n=1}^{m}(1+2 \alpha n) b^{2 n}-2 \sum_{n=2 m+3}^{\infty}(1+\alpha n) b^{n} .
\end{aligned}
$$

An induction shows that the right-hand side decreases with $m$, so that

$$
\begin{aligned}
\operatorname{Re} \tilde{h}^{\prime}(z) & \geq \frac{1-b^{2}-2 \alpha b}{(1+b)^{2}}-2 \sum_{n=1}^{\infty}(1+2 \alpha n) b^{2 n} \\
& =\frac{1-b^{2}-2 \alpha b}{(1+b)^{2}}-\frac{2 b^{2}\left(1+2 \alpha-b^{2}\right)}{\left(1-b^{2}\right)^{2}} \\
& =\frac{1}{1-b^{2}}\left[1-2 b-b^{2}-2 \alpha b\left(\frac{1+b^{2}}{1-b^{2}}\right)\right]=0
\end{aligned}
$$

CASE 4. We have

$$
\tilde{h}^{\prime}(z)=1+2 \sum_{n=1}^{m}\left[1+\alpha(2 n-1) e^{i \gamma}\right] z^{2 n-1}+2 \sum_{n_{k} \geq 2 m+3}\left[1+\alpha\left(n_{k}-1\right)\right] z^{n_{k}-1} .
$$

Then for $|z| \leq b$,

$$
\operatorname{Re} \tilde{h}^{\prime}(z) \geq 1-2 \sum_{n=1}^{m}[1+\alpha(2 n-1)] b^{2 n-1}-2 \sum_{n=2 m+3}^{\infty}[1+\alpha(n-1)] b^{n-1} .
$$

Again the right-hand side decreases with $m$ and

$$
\operatorname{Re} \tilde{h}^{\prime}(z) \geq 1-2 \sum_{n=1}^{\infty}[1+\alpha(2 n-1)] b^{2 n-1}=1-\frac{2 b}{1-b^{2}}-2 \alpha b\left(\frac{1+b^{2}}{\left(1-b^{2}\right)^{2}}\right)=0 .
$$

For sharpness, set $n_{k}=2 k$ so that $\tilde{f}(z)=z+2 \sum_{k=1}^{\infty} z^{2 k} / 2 k$. Setting $\gamma=0$ in (3.13), we see that $\tilde{h}^{\prime}(-b)=0$. 
4. Sufficient conditions. We next see how small the coefficient need to be in order to guarantee inclusion in the family.

THEOREM 4.1. A sufficient condition for $f$ of the form (1.1) to be in $\operatorname{UCD}(\alpha), \alpha \geq 0$, is that $\sum_{k=2}^{\infty} k[1+\alpha(k-1)]\left|a_{k}\right| \leq 1$.

Proof. Since Re $f^{\prime} \geq 1-\sum_{k=2}^{\infty} k\left|a_{k}\right|$ and $\left|z f^{\prime \prime}\right| \leq \sum_{k=2}^{\infty} k(k-1)\left|a_{k}\right|$, the result follows.

In [9] the family $T$ consisting of univalent functions $f$ of the form

$$
f(z)=z-\sum_{k=2}^{\infty} a_{k} z^{k}, \quad a_{k} \geq 0,
$$

was investigated. Denote by $\operatorname{TUCD}(\alpha)$ functions in $\operatorname{UCD}(\alpha)$ of the form (4.1). For this class, the sufficient condition of Theorem 4.1 is also necessary.

THEOREM 4.2. A function of the form (4.1) is in $\operatorname{TUCD}(\alpha)$ if and only if $\sum_{k=2}^{\infty} k[1+$ $\alpha(k-1)] a_{k} \leq 1$.

Proof. In view of Theorem 4.1, we need only show that $f \in \operatorname{TUCD}(\alpha)$ satisfies the coefficient condition. Note that

$$
f^{\prime}(r)=1-\sum_{k=2}^{\infty} k a_{k} r^{k-1}, \quad \alpha r f^{\prime \prime}(r)=\alpha \sum_{k=2}^{\infty} k(k-1) r^{k-1} .
$$

The result follows upon letting $r \rightarrow 1$.

REMARK 4.3. The coefficient characterizations found in [9] also show that $f$ of the form (4.1) is starlike $\Leftrightarrow f \in \operatorname{TUCD}(0)$, is convex $\Leftrightarrow f \in \mathrm{TUCD}(1)$, and is convex of order $1 / 2 \Leftrightarrow f \in \operatorname{TUCD}(2)$. A function $f$ of the form (4.1) is also uniformly convex $\Leftrightarrow f \in \operatorname{TUCD}(2)$. See [11].

From the work in [9], the coefficient characterization of Theorem 4.2 enables us to determine extreme points.

THEOREM 4.4. The extreme points of $\operatorname{TUCD}(\alpha)$ are $f_{1}(z)=z$ and

$$
f_{k}(z)=z-\frac{z^{k}}{k[1+\alpha(k-1)]}, \quad k=2,3, \ldots,
$$

and $f \in \operatorname{TUCD}(\alpha) \Leftrightarrow f$ can be expressed in the form

$$
f(z)=\sum_{k=1}^{\infty} \lambda_{k} f_{k}(z), \quad \text { where } \lambda_{k} \geq 0, \sum_{k=1}^{\infty} \lambda_{k}=1 .
$$

\section{REFERENCES}

[1] R. Fournier and S. Ruscheweyh, Remarks on a multiplier conjecture for univalent functions, Proc. Amer. Math. Soc. 116 (1992), no. 1, 35-43. MR 92k:30016. Zbl 848.30005.

[2] A. W. Goodman, On uniformly convex functions, Ann. Polon. Math. 56 (1991), no. 1, 87-92. MR 93a:30009. Zbl 744.30010. 
[3] D. J. Hallenbeck, Convex hulls and extreme points of some families of univalent functions, Trans. Amer. Math. Soc. 192 (1974), 285-292. MR 49\#3103. Zbl 296.30014.

[4] W. C. Ma and D. Minda, Uniformly convex functions, Ann. Polon. Math. 57 (1992), no. 2, 165-175. MR 93j:30009. Zbl 760.30004.

[5] T. H. MacGregor, Functions whose derivative has a positive real part, Trans. Amer. Math. Soc. 104 (1962), 532-537. MR 25\#4090. Zbl 106.04805.

[6] M. I. S. Robertson, On the theory of univalent functions, Ann. of Math. 37 (1936), 374-408. Zbl 014.16505.

[7] F. Rønning, Uniformly convex functions and a corresponding class of starlike functions, Proc. Amer. Math. Soc. 118 (1993), no. 1, 189-196. MR 93f:30017. Zbl 805.30012.

[8] S. Ruscheweyh, Extension of Szegö's theorem on the sections of univalent functions, SIAM J. Math. Anal. 19 (1988), no. 6, 1442-1449. MR 89m:30032. Zbl 661.30012.

[9] H. Silverman, Univalent functions with negative coefficients, Proc. Amer. Math. Soc. 51 (1975), no. 1, 109-116. MR 51\#5910. Zbl 311.30007.

[10] _ Generalized sequences for functions of positive real part, Houston J. Math. 19 (1993), no. 3, 421-428. MR 94j:30016. Zbl 792.30011.

[11] K. G. Subramanian, G. Murugusundaramoorthy, P. Balasubrahmanyam, and H. Silverman, Subclasses of uniformly convex and uniformly starlike functions, Math. Japon. 42 (1995), no. 3, 517-522. MR 96h:30018. Zbl 837.30011.

Rosy, StePhen, and Subramanian: DePartment of Mathematics, Madras Christian COLLEgE, TAMBARAM, MADRAS-600 059, INDIA

Silverman: Department of Mathematics, College of Charleston, Charleston, SC 29424, USA

E-mail address: si 1vermanh@cofc.edu 


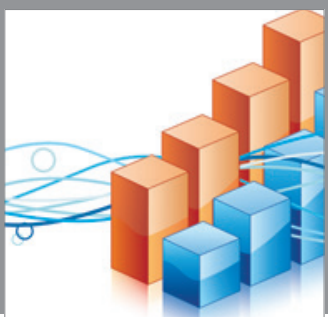

Advances in

Operations Research

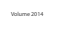

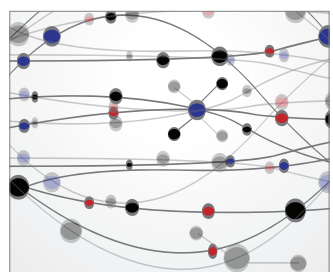

\section{The Scientific} World Journal
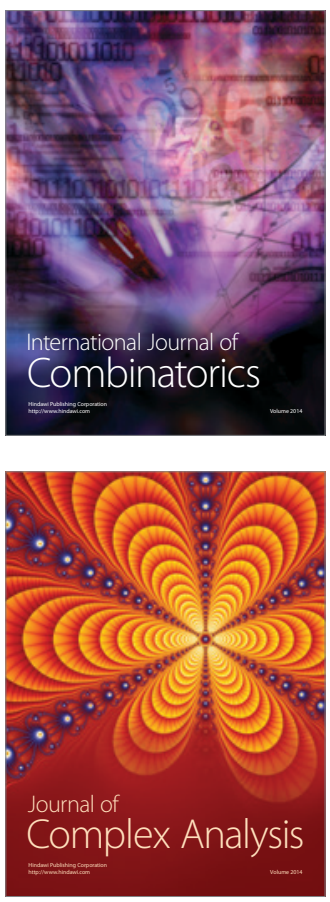

International Journal of

Mathematics and

Mathematical

Sciences
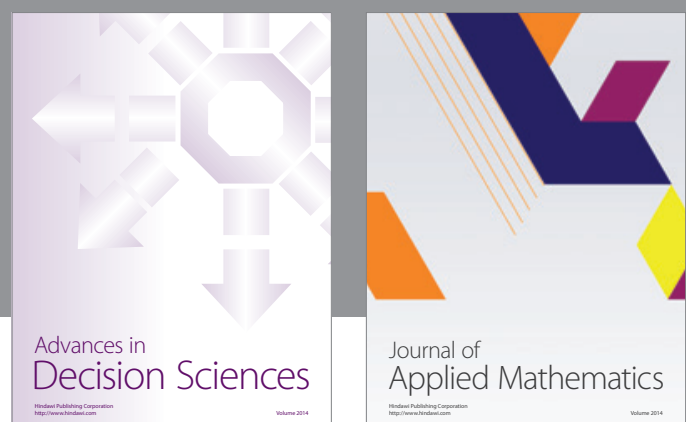

Journal of

Applied Mathematics
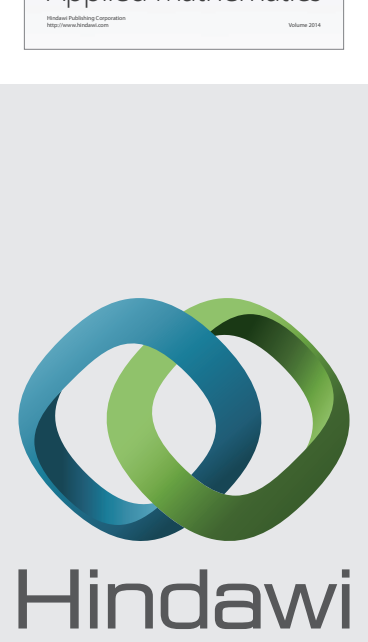

Submit your manuscripts at http://www.hindawi.com
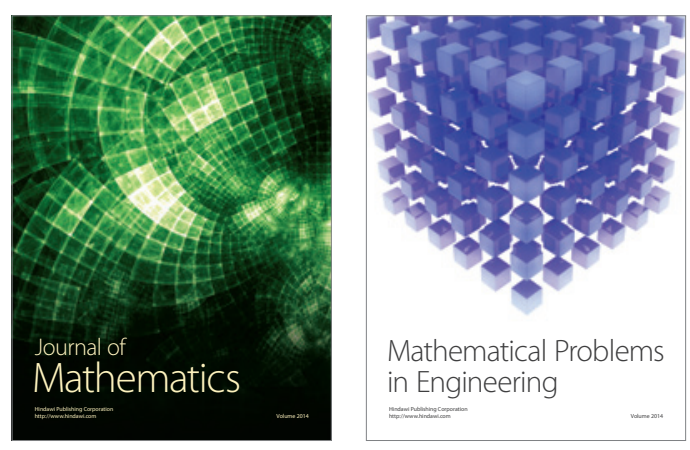

Mathematical Problems in Engineering
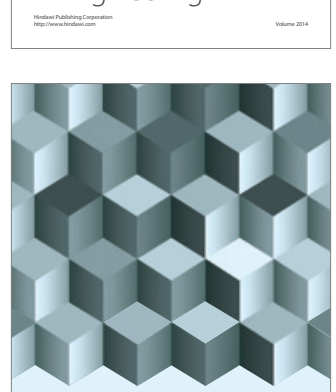

Journal of

Function Spaces
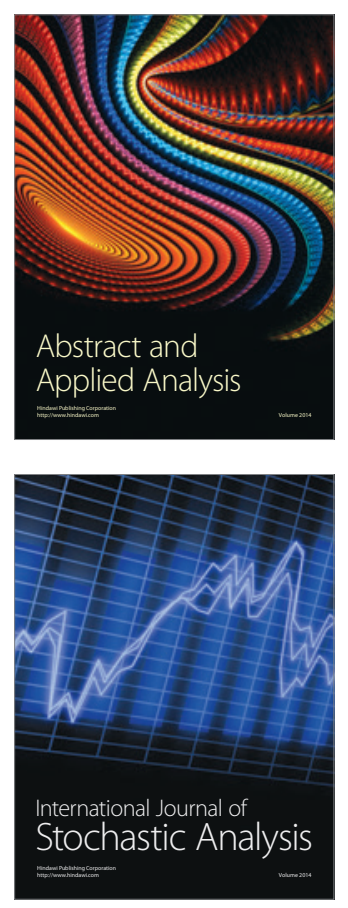

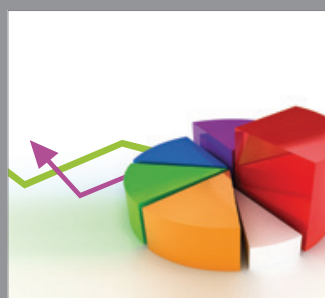

ournal of

Probability and Statistics

Promensencen
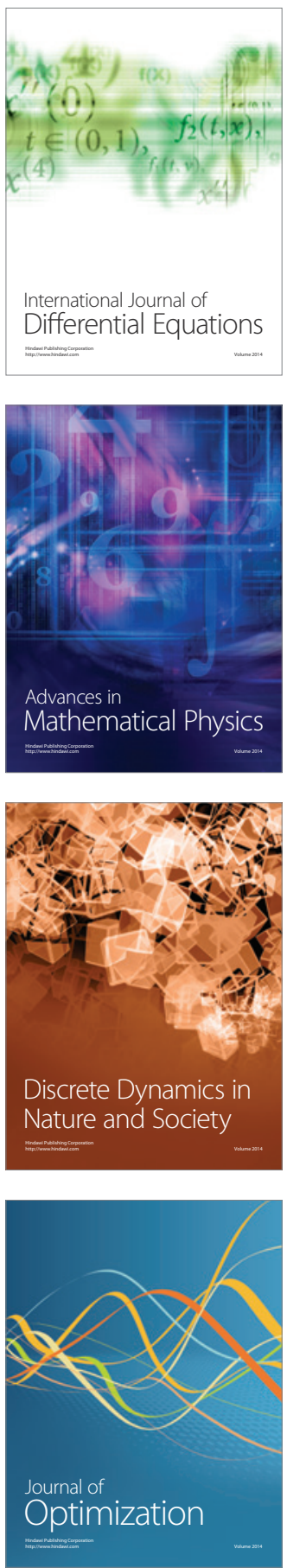